\title{
Correlation between Clinical Outcomes and Baseline CT and CT Angiographic Findings in the SWIFT PRIME Trial
}

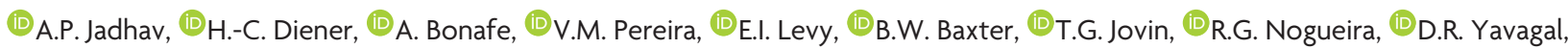

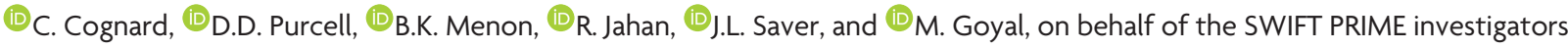

\begin{abstract}
BACKGROUND AND PURPOSE: Patient selection for endovascular therapy remains a great challenge in clinic practice. We sought to determine the effect of baseline CT and angiography on outcomes in the Solitaire With the Intention for Thrombectomy as Primary Endovascular Treatment for Acute Ischemic Stroke (SWIFT PRIME) trial and to identify patients who would benefit from endovascular stroke therapy.
\end{abstract}

MATERIALS AND METHODS: The primary end point was a 90-day modified Rankin Scale score of 0-2. Subgroup and classification and regression tree analysis was performed on baseline ASPECTS, site of occlusion, clot length, collateral status, and onset-to-treatment time.

RESULTS: Smaller baseline infarct $(n=145)$ (ASPECTS 8-10) was associated with better outcomes in patients treated with thrombectomy versus IV tPA alone (66\% versus $41 \%$; rate ratio, 1.62 ) compared with patients with larger baseline infarcts $(n=44)$ (ASPECTS $6-7)$ ( $42 \%$ versus $21 \%$; rate ratio, 1.98). The benefit of thrombectomy over IV IPA alone did not differ significantly by ASPECTS. Stratification by occlusion location also showed benefit with thrombectomy across all groups. Improved outcomes after thrombectomy occurred in patients with clot lengths of $\geq 8 \mathrm{~mm}$ ( $71 \%$ versus $43 \%$; rate ratio, 1.67). Outcomes stratified by collateral status had a benefit with thrombectomy across all groups: none-fair collaterals (33\% versus $0 \%$ ), good collaterals ( $58 \%$ versus $44 \%$ ), and excellent collaterals ( $82 \%$ versus $28 \%$ ). Using a 3-level classification and regression tree analysis, we observed optimal outcomes in patients with favorable baseline ASPECTS, complete/ near-complete recanalization ( $\mathrm{TICl} 2 \mathrm{~b} / 3$ ), and early treatment (mean mRS, 1.35 versus 3.73), while univariate and multivariate logistic regression showed significantly better results in patients with higher ASPECTS.

CONCLUSIONS: While benefit was seen with endovascular therapy across multiple subgroups, the greatest response was observed in patients with a small baseline core infarct, excellent collaterals, and early treatment.

$\mathbf{P}$ atient selection for mechanical thrombectomy in acute ischemic strokes presents a major challenge in achieving good outcomes. First-generation randomized controlled trials investi-

Received April 3, 2017; accepted after revision July 19.

From Department of Neurology and Neurological Surgery (A.P.J., T.G.J.), University of Pittsburgh Medical Center, Pittsburgh, Pennsylvania; Department of Neurology (H.-C.D,), University Hospital of University Duisburg-Essen, Essen, Germany; Department of Neuroradiology (A.B.), Hôpital Gui-de-Chauliac, Montpellier, France; Division of Neuroradiology and Division of Neurosurgery (V.M.P.), Toronto Western Hospital, University Health Network, University of Toronto, Ontario, Canada; Department of Neurosurgery (E.I.L.), State University of New York at Buffalo, Buffalo, New York; Department of Radiology (B.W.B.), Erlanger Hospital at University of Tennessee, Chattanooga, Tennessee; Department of Neurology (R.G.N.), Marcus Stroke and Neuroscience Center, Grady Memorial Hospital, Emory University School of Medicine, Atlanta, Georgia; Department of Neurology and Neurosurgery (D.R.Y.), University of Miami Miller School of Medicine/Jackson Memorial Hospital, Miami, Florida; Department of Diagnostic and Therapeutic Neuroradiology (C.C.), University Hospital of Toulouse, Toulouse, France; BioClinica (D.D.P.), Newark, California; Department of Radiology (D.D.P.), California Pacific Medical Center, San Francisco, California; Division of Neuroradiology (D.D.P.), University of California San Francisco, San Francisco, California; Departments of Radiology and Clinical Neurosciences (M.G.) and Neurology and Neurosurgery (B.K.M.), University of Calgary, Calgary, Alberta, Canada; and Division of Interventional Neuroradiology (R.J.) and Department of Neurology and Comprehensive Stroke Center (J.L.S.), David gating the benefit of intra-arterial therapy failed to demonstrate improved rates of independence in the treatment group. A limitation of these trials was the large baseline core infarcts at the time of enrollment. In the Interventional Management of Stroke III (IMS III) trial, $40 \%$ of patients had lower Alberta Stroke Program Early CT Scores on presentation (ASPECTS 0-7). ${ }^{1}$ In patients with lower ASPECTS, there was a 2-fold less likelihood of benefit with IV or intra-arterial therapy compared with patients with higher ASPECTS. In the Mechanical Retrieval and Recanalization

Geffen School of Medicine at the University of California Los Angeles, University of California, Los Angeles, Los Angeles, California.

An academic steering committee supervised the trial design and operations. The sponsor of the study (Covidien) was responsible for site management, data management, and safety reporting. The study data were independently monitored. The statistical analyses were prepared by an independent external statistician (S. Brown; Altair Biostatistics, St. Louis Park, Minnesota).

This work was supported by the SWIFT PRIME study, funded by Covidien.

Please address correspondence to Mayank Goyal, MD, Department of Radiology, University of Calgary, Foothills Hospital, 1403 29th St NW, Calgary, AB, Canada; e-mail: mgoyal@ucalgary.ca

http://dx.doi.org/10.3174/ajnr.A5406 
of Stroke Clots Using Embolectomy (MR RESCUE) trial, the core baseline infarct was $36 \mathrm{~mL}$ at enrollment with only $21 \%$ of patients achieving functional independence at 90 days (modified Rankin Scale score, $0-2){ }^{2}$ To overcome these constraints, the Solitaire With the Intention for Thrombectomy as Primary Endovascular Treatment for Acute Ischemic Stroke (SWIFT PRIME) trial limited enrollment to patients with small-moderate core infarcts as defined by head CT, CT angiography, and/or CT perfusion. We have previously reported on the primary outcomes of the SWIFT PRIME trial ${ }^{3}$ and the secondary prespecified analysis of baseline CT perfusion imaging and follow-up infarct volume and outcomes. ${ }^{4,5}$ In this study, we describe the effect of the baseline CT and CTA findings on clinical outcome.

\section{MATERIALS AND METHODS}

The study design of the SWIFT PRIME trial has been previously described. ${ }^{3,6}$ Primary outcomes for all 196 patients have been previously reported, ${ }^{3}$ while a subset of 151 patients has been evaluated and presented for outcomes based specifically on perfusion imaging. ${ }^{4}$ In this article, we report, for the first time, the effects of imaging parameters (including ASPECTS and collateral status), substantial reperfusion, and time to treatment on clinical outcomes. An independent core imaging lab evaluated all imaging. Baseline head CT was available for review in 185 patients. CT angiography was available for review in $88 \%$ of patients. The ASPECTS is a 10-point semiquantitative topographic score for assessing stroke burden in the middle cerebral artery distribution on CT. ${ }^{7}$ Enrollment (after the first 71 patients) was restricted to patients with ASPECTS scores of $>5$. For the first 71 patients, the inclusion criteria were based on a CT perfusion study as follows: ischemic core lesion volume, $\leq 50 \mathrm{~mL}$; time-to-maximum, $>10$ seconds; lesion volume, $\leq 100 \mathrm{~mL}$; mismatch volume, $\geq 15 \mathrm{~mL}$; and mismatch ratio, $>1.8$. Ischemic core was defined as an area with $>70 \%$ reduction in $\mathrm{CBF}$ (relative $\mathrm{CBF}<0.3$ ) in comparison with the mean $\mathrm{CBF}$ of normally perfused brain parenchyma. An ischemic core lesion defined by CT perfusion corresponds to an ASPECTS of $>5$.

For subgroup analysis according to ASPECTS, we made 2 comparisons of higher-versus-lower ASPECTS: ASPECTS 8-10 versus ASPECTS 6-7 and ASPECTS 9-10 versus ASPECTS 6-8. The site of occlusion was defined by baseline head CTA: ICA occlusion, proximal $\mathrm{M} 1$ occlusion, middle M1 occlusion, and distal M1/M2 occlusion. Clot length was measured on CTA or MRA as the length of a vessel that was nonopacified/nonvisualized using 5-mm multiplanar MIP reformations. Contrast-enhanced MRA was used whenever possible. In a subset of cases in which the distal end of the clot could not be identified, CT perfusion source images allowed visualization and measurement of the clot. The earlier phases of CTP were used to determine the proximal end of the clot, while the later phases were used to determine the distal end of the clot. Prior studies have identified a thrombus length of $\geq 8 \mathrm{~mm}$ in the middle cerebral artery as being refractory to recanalization from intravenous thrombolysis, ${ }^{8}$ which can potentially impact clinical outcomes. To understand the impact of clot length on responsiveness to endovascular therapy, clinical outcomes were compared in patients with $\geq 8 \mathrm{~mm}$ of thrombus.

\section{Collateral Scoring on CTA}

Collateral assessment was defined on CTA as excellent, good, fair, poor, minimal, or none. ${ }^{9}$ Definitions were as follows: excellent, increased or normal prominence and extent of pial vessels beyond the occluded artery within the symptomatic hemisphere; good, slightly reduced prominence and extent of pial vessels beyond the occluded artery within the symptomatic hemisphere; fair, moderately reduced prominence and extent of pial vessels beyond the occluded artery within the symptomatic hemisphere; poor, decreased prominence and extent and regions with no vessels in some part of the occluded territory; minimal, compared with the asymptomatic contralateral hemisphere, just a few vessels visible in the occluded vascular territory; and none, no vessels visible within the occluded vascular territory. ${ }^{9}$

\section{Statistical Analysis}

All available data were used for analyses. Statistical tests for binary variables were performed with the Fisher exact test, and for continuous variables, they were performed with the Student $t$ test. Univariate and multivariate logistic regressions were used to test relationships between potential predictor variables and outcomes defined by the modified Rankin Scale score of $0-2$ at 90 days. Classification and regression tree analysis were used to further investigate relationships among study variables. All statistical tests were 2 -sided. with $P$ values $<.05$ considered statistically significant. All analyses were performed in R, Version 3.0 or above (R Foundation for Statistical Computing; Vienna, Austria; http:// www.R-project.org).

\section{RESULTS}

\section{Baseline CT ASPECTS}

A higher baseline ASPECTS of 8-10 was noted in 145 patients (74 in the IV tPA arm; 71 in the endovascular and IV tPA arm), of whom 142 had mRS available at 90 days. Good outcomes (mRS $0-2)$ at 90 days were observed in $66 \%$ of patients in the treatment arm compared with $41 \%$ of patients in the control arm $(P=.004)$. Lower baseline ASPECTS of 6-7 was noted in 44 patients (24 in the IV tPA arm; 20 in the endovascular and IV tPA arm). Good outcomes were observed in $42 \%$ of patients in the treatment arm compared with $21 \%$ of patients in the control arm $(P=.2$; Fig 1$)$. In univariate and multivariate logistic regression analyses, a higher baseline ASPECTS was associated with better outcomes, particularly when dichotomized for ASPECTS of 9-10 versus $\leq 8$ (Tables 1 and 2).

\section{Site of Occlusion}

Distribution of the site of occlusion was as follows: ICA (20 patients), proximal M1 (39 patients), middle M1 (55 patients), and distal M1/M2 (49 patients). Treatment effect with endovascular therapy was noted across all sites of occlusion (Fig 2), with the greatest treatment effect in patients with a proximal M1 occlusion ( $88 \%$ versus $14 \%, P<.0001$ ). The site of occlusion was not significantly associated with good outcome in the univariate analysis (Table 1).

AJNR Am J Neuroradiol 38:2270-76 Dec 2017 www.ajnr.org 2271 


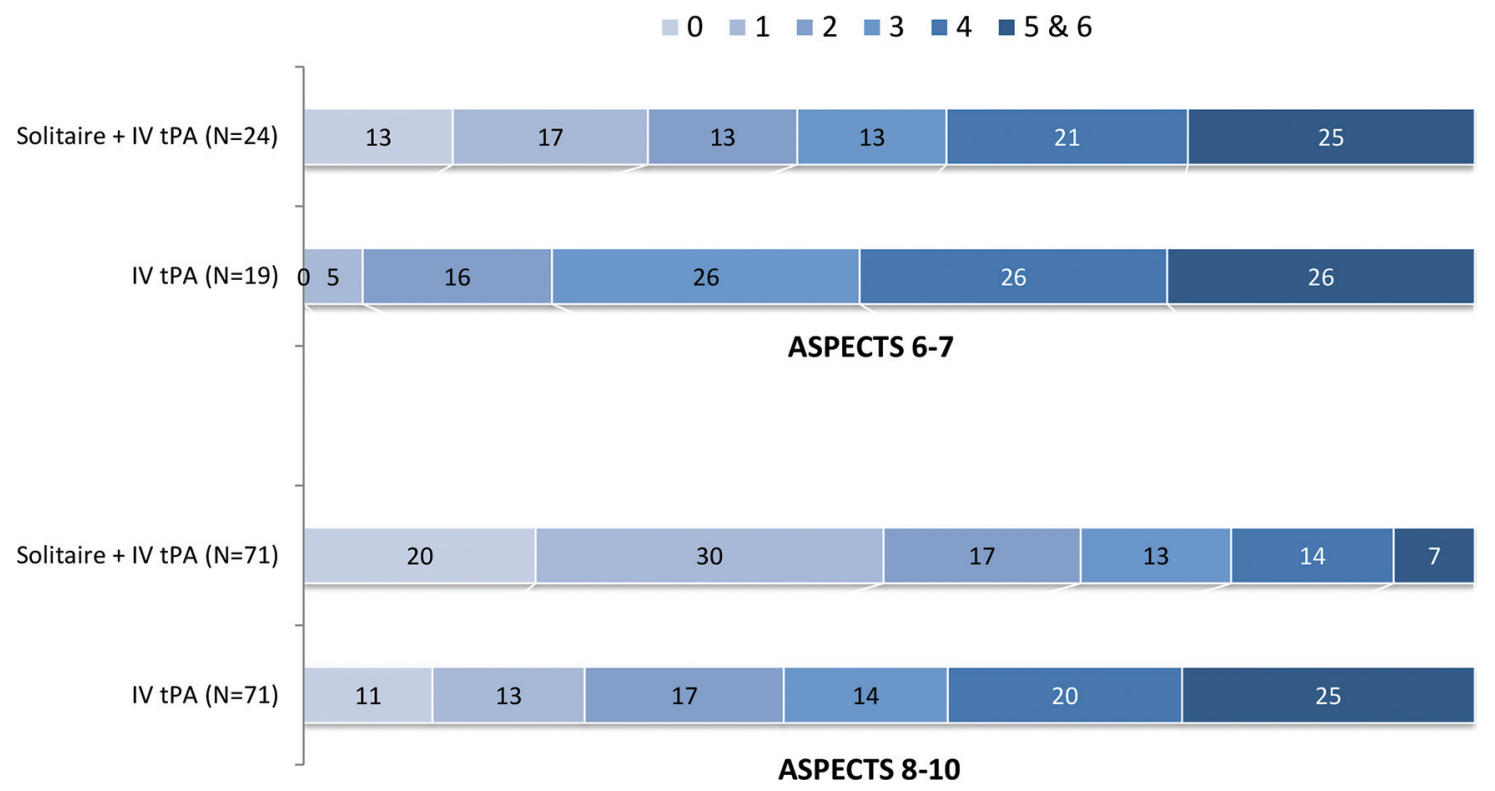

FIG 1. Influence of baseline CT ASPECTS on outcome (90-day mRS), indicating better outcomes with higher ASPECTS and better outcome with endovascular therapy irrespective of ASPECTS.

Table 1: Univariate predictors of functional independence (mRS 0-2) at 90 days, endovascular arm only

\begin{tabular}{|c|c|c|c|c|c|c|}
\hline Predictor & No. Total & No. Category & Odds Ratio & Lower $\mathrm{Cl}$ & Upper Cl & $P$ Value \\
\hline ASPECTS (per unit) & 95 & NA & 1.53 & 1.09 & 2.15 & .015 \\
\hline ASPECTS 8-10 (vs 6-7) & 95 & $71 / 24$ & 2.74 & 1.06 & 7.08 & .037 \\
\hline ASPECTS 9-10 (vs 6-8) & 95 & $52 / 43$ & 3.43 & 1.45 & 8.09 & .005 \\
\hline Ml occlusion (vs non-M1) & 95 & $60 / 35$ & 2.12 & 0.90 & 4.97 & .085 \\
\hline Onset-to-groin puncture (per $60 \mathrm{~min}$ ) & 94 & NA & 0.68 & 0.48 & 0.98 & .040 \\
\hline $\mathrm{TICl} 3$ postprocedure (vs $\leq \mathrm{TICl} 2 \mathrm{~b}$ ) & 80 & $56 / 24$ & 2.11 & 0.79 & 5.61 & .13 \\
\hline $\mathrm{TICl} 2 \mathrm{~b} / 3$ postprocedure (vs $\leq \mathrm{TICI} 2 \mathrm{a}$ ) & 80 & $70 / 10$ & 1.80 & 0.47 & 6.82 & .39 \\
\hline Collateral grade (per unit) & 56 & NA & 2.77 & 1.13 & 6.82 & .026 \\
\hline Clot length (per mm) & 50 & NA & 1.12 & 0.96 & 1.30 & .14 \\
\hline Clot length of $\geq 8 \mathrm{~mm}$ (vs $<8 \mathrm{~mm}$ ) & 50 & $44 / 6$ & 2.38 & 0.42 & 13.40 & .32 \\
\hline Clot length of $\geq 13 \mathrm{~mm}$ (vs $<13 \mathrm{~mm}$ ) & 50 & $23 / 27$ & 2.48 & 0.71 & 8.66 & .16 \\
\hline
\end{tabular}

Note:-No. reflects the number of data points available for each variable; NA, not applicable.

${ }^{a}$ Univariate logistic regression analysis of patients undergoing endovascular therapy was performed with the following variables: ASPECTS (as scored on preprocedural head CT, $6-7$ vs $8-10$ and $6-8$ vs $9-10$ ), site of occlusion (M1 versus non-M1), onset to groin puncture, quality of recanalization ( $\mathrm{TICl}$ scale), collateral grade, and clot length ( $>8 \mathrm{~mm}$ versus $<8 \mathrm{~mm})$.

Table 2: Multivariate predictors of functional independence (mRS 0-2) at 90 days, endovascular arm only ${ }^{\mathrm{a}}$

\begin{tabular}{lcccccc}
\hline \multicolumn{1}{c}{ Predictor } & No. & No. & Odds & Lower & Upper & $\begin{array}{c}\boldsymbol{P} \\
\text { Category }\end{array}$ \\
& Ratio & Cl & Cl & Value \\
\hline ASPECTS 9-10 (vs 6-8) & 48 & $28 / 20$ & 4.25 & 1.06 & 17.10 & .042 \\
Onset-to-groin puncture (per 60 min) & 48 & NA & 0.79 & 0.44 & 1.42 & .43 \\
TICI 2b/3 postprocedure (vs $\leq \mathrm{TICl}$ 2a) & 48 & $43 / 5$ & 1.03 & 0.12 & 8.46 & .98 \\
Collateral grade (per unit) & 48 & NA & 2.85 & 0.90 & 9.08 & .076 \\
\hline
\end{tabular}

Note:-No. reflects the number of data points available for each variable; NA, not applicable.

a Multivariate logistic regression analysis of patients undergoing endovascular therapy was performed with the following variables: ASPECTS (as scored on preprocedural head CT), onset to groin puncture, quality of recanalization (TICI scale), and collaterals.

\section{Length of Clot}

Clot length was available in 111 patients, of whom $89 \%$ had $\geq 8$-mm clot length. In this subgroup, $71 \%$ of patients having undergone thrombectomy versus $43 \%$ of patients receiving IV tPA alone had good outcomes $(P=.005$, Fig 3$)$. Median clot length was $13 \mathrm{~mm}$. In 59 patients with a clot length greater than the median, $79 \%$ of patients having undergone thrombectomy versus $34 \%$ of patients with IV tPA alone had good outcomes ( $P=$ .001). In univariate analysis, clot length was not associated with good outcome in the subgroup of patients treated with endovascular therapy (Table 1).

\section{Quality of Collaterals}

Collateral status on baseline head CTA was available for review in 113 patients. Poor collaterals (none-to-fair) were noted in $19 \%$ of patients with a median baseline ASPECTS of 8 and a mean core infarct volume of $18.9 \mathrm{~mL}$. High-quality collaterals (good-to-excellent) were associated with a median baseline ASPECTS of 9 and a mean core infarct volume of 7.4 $\mathrm{mL}$. A beneficial effect of endovascular therapy was observed over IV tPA alone across all levels of collateral flow, with the greatest effect in patients with excellent collaterals ( $82 \%$ versus $28 \%, P=.008$; Fig 4 ). In univariate and multivariate analysis, a good collateral grade was associated with good outcome (statistically significant in univariate analysis and a trend toward significance in multivariate analysis) (Tables 1 and 2). 


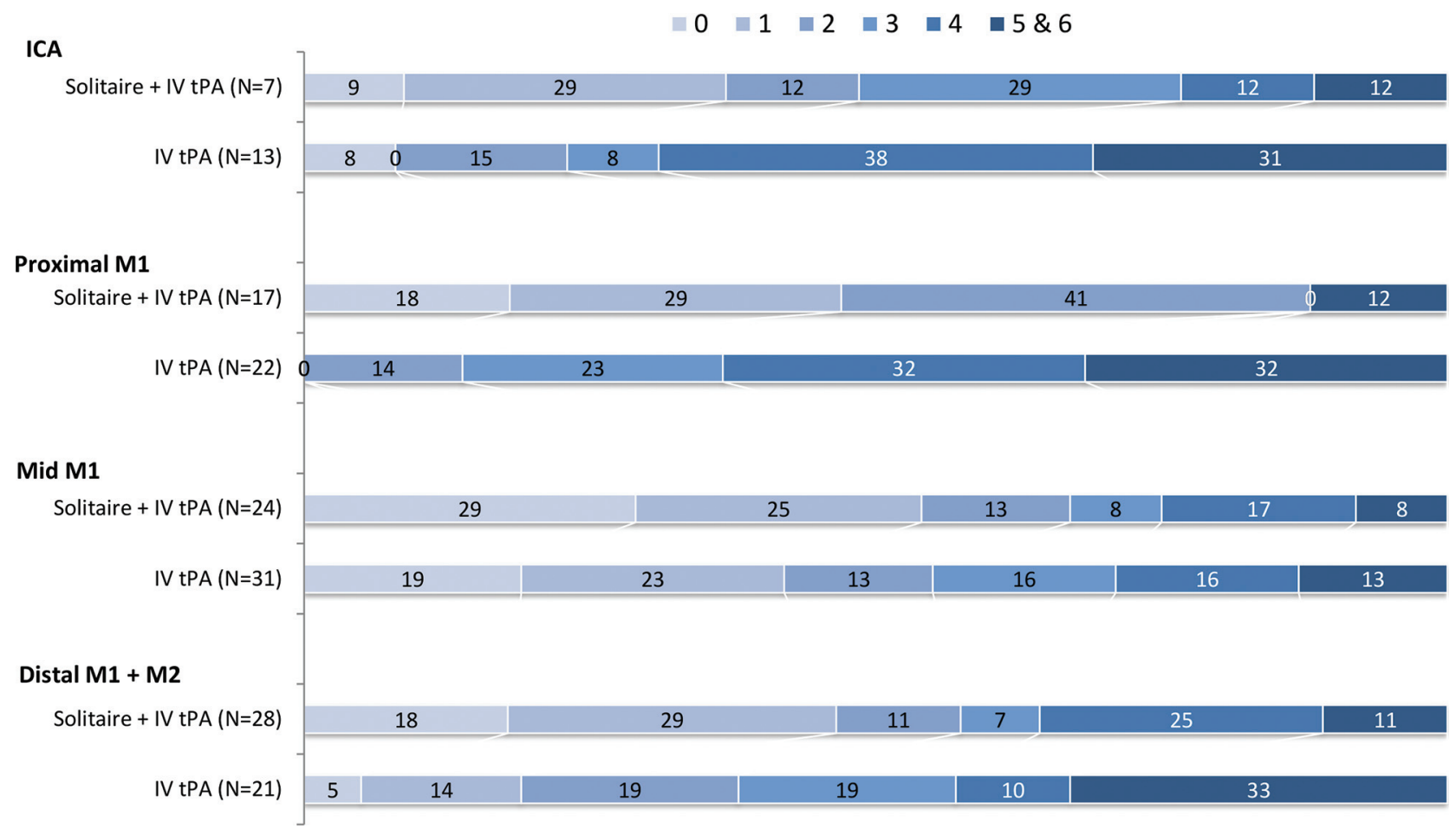

FIG 2. Influence of the site of occlusion on outcome (90-day mRS), indicating better outcome with endovascular therapy irrespective of site and the greatest treatment effect in proximal Ml occlusions.

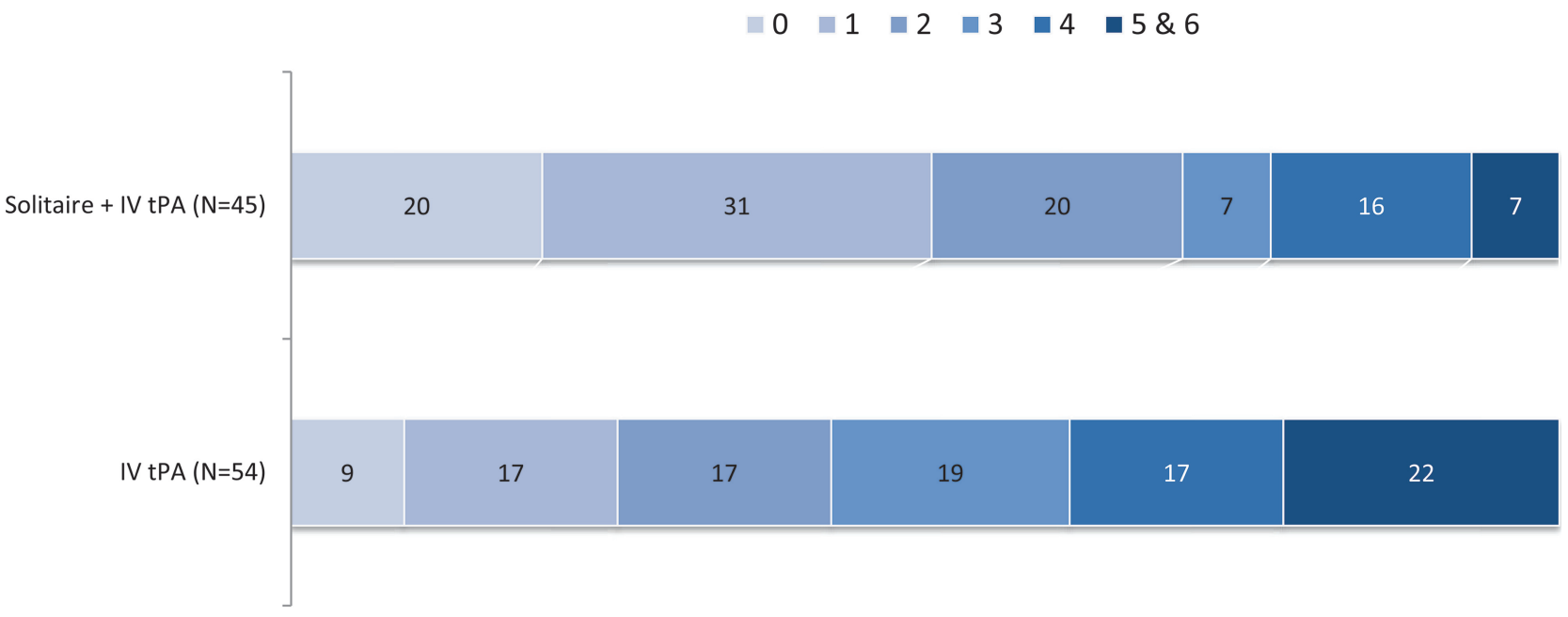

FIG 3. Outcomes (90-day $\mathrm{mRS}$ ) in patients with a clot length of $\geq 8 \mathrm{~mm}$, indicating statistically superior outcomes with endovascular therapy in these clots.

\section{Classification and Regression Tree Analysis}

Univariate analysis revealed that favorable ASPECTS, earlier treatment, good collaterals, and complete recanalization are associated with better outcomes (Table 1). With the classification and regression tree algorithm, binary cut points affecting outcome were identified as dichotomized ASPECTS (ASPECTS 9-10 versus ASPECTS 6-8), onset-to-puncture time (within 4 hours versus beyond 4 hours), and quality of recanalization (TICI 3 versus TICI $2 \mathrm{~b}$ or less). Of 98 patients undergoing thrombectomy, better outcomes were observed in patients with favorable ASPECTS, early treatment, and complete recanalization (Fig 5). In the patients undergoing thrombectomy, the average time from symp- tom onset to recanalization was 260 minutes and the rate of TICI $2 \mathrm{~b} / 3$ recanalization was $88 \%(70 / 80)$.

\section{DISCUSSION}

While the primary results of the SWIFT PRIME study revealed a benefit of endovascular therapy over intravenous thrombolysis alone in patients with acute ischemic stroke, several questions remain about understanding subsets of patients most likely to benefit from intra-arterial therapy. In this report, we found that the benefit of endovascular therapy persisted across multiple subgroups, with the highest likelihood of benefit noted in patients with higher ASPECTS, early treatment, and favorable collaterals. 


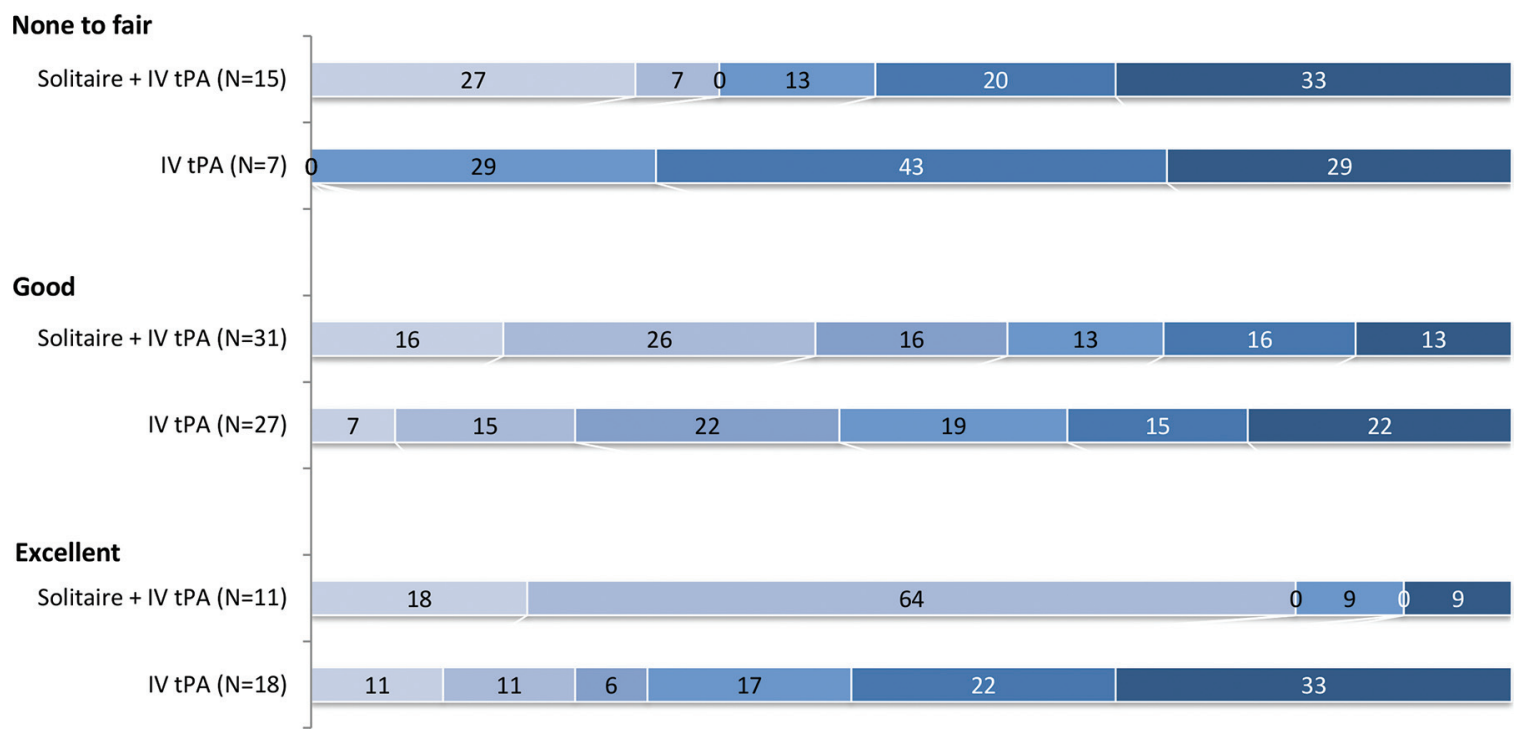

FIG 4. Influence of baseline collateral status on outcome (90-day mRS), indicating better outcomes with better collaterals, better outcomes with endovascular therapy irrespective of collateral quality, and the greatest treatment effect associated with excellent collaterals.

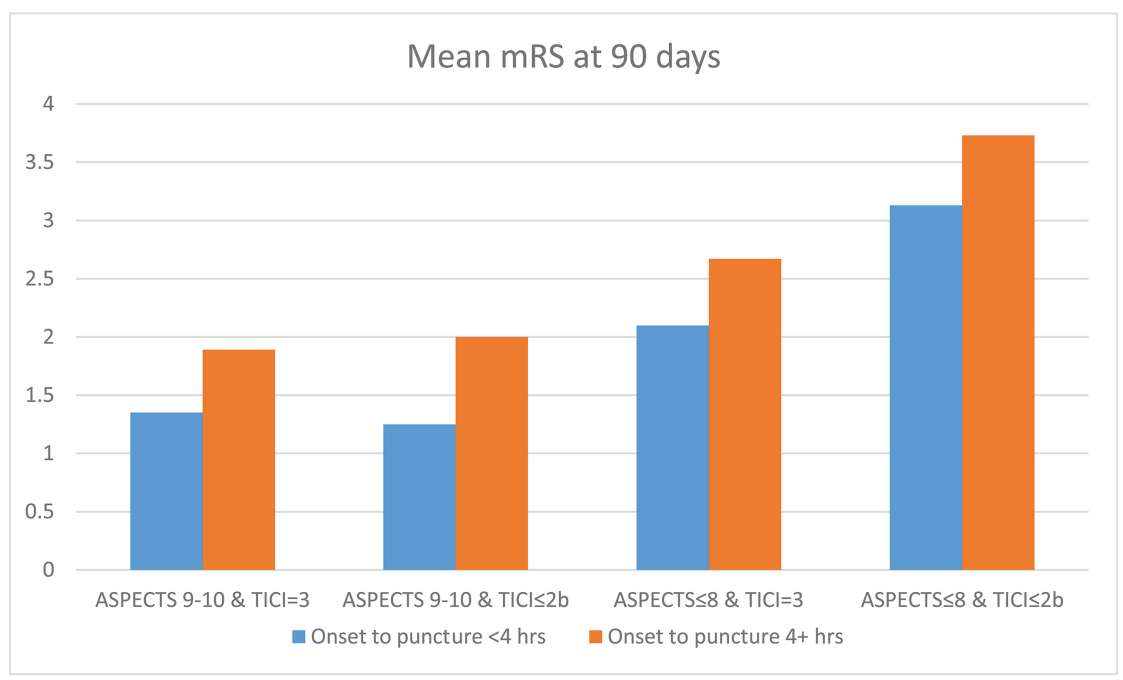

FIG 5. Mean mRS in dichotomized subgroups identified by classification and regression tree analysis indicating time to recanalization, initial ASPECTS, and TICI scores posttreatment as substantial predictors of outcome with the displayed cut-points for dichotomization.

In the SWIFT PRIME trial, patients were selected for enrollment on the basis of small core infarcts on presentation. Accordingly, the current analysis is limited to patients with overall favorable ASPECTS. The benefit of complete or near-complete recanalization of patients with poor ASPECTS $(<5)$ remains unclear and may warrant further investigation, ${ }^{12}$ particularly in younger patients in whom recanalization may limit further infarct growth. Such a clinical result may not be well-captured in a dichotomized mRS outcome of $0-2$ versus 3-6, but it may spare a young patient hemicraniectomy, respiratory compromise, or other stroke-related complications. Furthermore, a population with a poor ASPECTS may be well-suited for bridging neuroprotection therapies in which recanalization along with adjunctive ther-

Previous studies of endovascular therapy (Intra-arterial Prourokinase for Acute Ischemic Stroke [PROACT II] and IMSI) demonstrated a strong interaction between favorable ASPECTS $(8-10)^{10,11}$ and outcome; however, a similar analysis of IMS III did not confirm this relationship. ${ }^{1}$ Failure to appreciate this relationship in IMS III has been attributed to long onset-to-treatment times as well as the low reperfusion rates in the treatment arm. In contrast, the SWIFT PRIME trial had fast treatment times and high rates of reperfusion. Furthermore, the baseline ASPECTSs in SWIFT PRIME were much higher, with $72 \%$ of patients having an ASPECTS of 8-10 compared with $58 \%$ of patients in IMS III. ${ }^{1}$ We found that patients with higher ASPECTS had better clinical outcomes, particularly with endovascular therapy. This finding is in keeping with numerous other studies demonstrating that baseline ASPECTS is an important predictor of final outcome. apy may result in acceptable outcomes. ${ }^{13}$

ASPECTS alone is a single freeze-frame in the evolution of necrosing brain tissue. Additional information about the speed and extent of infarct burden can be inferred by clinical examination (a large deficit suggests large tissue at risk), perfusion imaging, and collateral status. The presence of robust collateral blood circulation indicates brain tissue that is more likely to be reperfused and, when reperfused, more likely to have a favorable response. ${ }^{14}$ Additionally, patients with robust collaterals are likely to have smaller core infarcts on presentation. Furthermore, previous studies have indicated that reperfusion therapies in patients with poor baseline collateral circulation do not typically have a favorable response, and this feature eventually results in a higher likelihood of infarct growth. ${ }^{15}$ Most interesting, we found trends toward benefit in patients with poor baseline collateral status after 
endovascular therapy, though the overall numbers are small and not statistically significant. This population will require further examination because the natural history tends to be quite poor and treatment options are limited. A caveat with collateral assessment is that single-phase CTAs may mislabel patients with moderate-to-good collaterals as poor in CTAs that are mistimed (acquired in the early arterial phase). Collateral assessment with multiphase CTA is a potential solution. ${ }^{16}$ Arguably, patients with poor collateral circulation presenting at early time windows may continue to benefit from thrombectomy if achieved in ultrarapid fashion. Given the quickly growing core infarct in this population, there may be a role for additional therapies designed to arrest stroke progression, such as neuroprotective therapy ${ }^{17}$ or hypothermia.

The site of occlusion and thrombus burden is associated with failure of IV tPA to recanalize large-vessel occlusion. Distal occlusions such as M2 or distal M1 are particularly responsive to IV tPA, whereas proximal occlusions such as ICA or proximal M1 are more refractory to IV tPA. ${ }^{18}$ While benefit was observed with endovascular therapy across groups, the highest benefit was noted in proximal M1 occlusions compared with distal M1 occlusions. Similarly, IV tPA is less effective as thrombus burden increases. In 1 study of patients undergoing intravenous thrombolysis for acute stroke, hardly any patients $(<1 \%)$ with clot measuring $\geq 8$ $\mathrm{mm}$ had successful recanalization. ${ }^{8}$ One limitation of measuring clot length on CTA is that it does not accurately define the thrombus extent because the lack of distal contrast opacification may be related to delayed distal filling rather than a true filling defect. In univariate analysis, the site of occlusion and clot length did not predict 90-day mRS 0-2 after endovascular therapy. It is possible that the effectiveness of endovascular therapy to recanalize such clots may mitigate the role of clot length in predicting good clinical outcomes. A priori identification of IV tPA nonresponders may ultimately guide future management strategies in which IV thrombolysis may be bypassed in favor of a direct intra-arterial therapy. ${ }^{18}$

\section{Limitations}

There are important limitations to our study. First, the sample size is relatively small, so further validation of our findings will require analysis in a larger cohort of patients. Second, given the nature of the study design and focus on patients re-presenting with small core infarcts, very few patients in our analysis had large core infarcts on initial presentation. The extent of benefit in this larger core population remains unanswered. Additionally, the participating clinical sites in the SWIFT PRIME trial were specifically selected on the basis of clinical volume and expertise. The generalizability of these results across additional centers remains untested. Finally, this study included post hoc analysis, so additional confirmation will require prospective studies of specific subgroups and patient features.

\section{CONCLUSIONS}

Overall, this report supports the selection of patients for intraarterial therapy on the basis of favorable patient characteristics (small core, good collateral circulation) and low likelihood of recanalization with intravenous thrombolysis (large and proximal clot burden). Additional studies will be needed to further understand the continued benefit of intra-arterial treatment for patients with larger infarct burden or distal occlusions.

\section{ACKNOWLEDGMENTS}

The authors thank Scott Brown for statistical analysis, Mark Lowerison for help with figure design, and Cynthia Yang and Rachel Castro for project management.

Disclosures: Hans-Christoph Diener-OTHER RELATIONSHIPS: H.-C.D. received honoraria for participation in clinical trials, contribution to advisory boards, or oral presentations from the following: Abbott, Allergan, AstraZeneca, Bayer Vital, BristolMyers Squibb, Boehringer Ingelheim, CoAxia, Corimmun, Covidien, Daiichi Sankyo, D-Pharm, Fresenius, GlaxoSmithKline, Janssen-Cilag, Johnson \& Johnson, Knoll, Eli Lilly, Merck Sharp \& Dohme, Medtronic, Mindframe, Neurobiological Technologies, Novartis, Novo Nordisk, Paion, Parke-Davis, Pfizer, Sanofi-Aventis, Schering-Plough, Servier, Solvay, St. Jude, Syngis, Talecris Pharma Resources, Thrombogenics, WebMD Global, Wyeth and Yamanouchi. Financial support for research projects was provided by AstraZeneca, GlaxoSmithKline, Boehringer Ingelheim, Lundbeck, Novartis, Janssen-Cilag, Sanofi-Aventis, Syngis, and Talecris Pharma Resources. The Department of Neurology at the University Duisburg-Essen received research grants from the German Research Council, German Ministry of Education and Research, European Union, National Institutes of Health, the Bertelsmann Foundation, and the Heinz-Nixdorf Foundation. H.-C.D. has no ownership interest and does not own stocks in any pharmaceutical company. Alain Bonafe-UNRELATED: Consultancy: Medtronic, Stryker. Vitor Mendes Pereira-RELATED: Consulting Fee or Honorarium: Medtronic/Covidien, Comments: as EU Interventional Principal Investigator for the SWIFT PRIME trial*; UNRELATED: Consultancy: Medtronic, Stryker, Codman Neuro, Penumbra, Comments: proctoring and studies Advisory Board. Elad I. LevyUNRELATED: Board Membership: Stryker, NeXtGen Biologics, MEDX, Cognition Medical Corp, Comments: serves on the Advisory Board; Consultancy: Pulsar Vascular; Expert Testimony, Comments: renders medical/legal opinion as an expert witness; Payment for Development of Educational Presentations: Covidien, Comments: honorarium for training and lectures; Stock/Stock Options: Intratech Medical, NeXtGen Biologics, Comments: shareholder/ownership interest; Other: Abbott Vascular, Comments: carotid training sessions for physicians. Blaise W. BaxterUNRELATED: Consultancy: Penumbra, Comments: consulting fees for stroke therapy development; Payment for Lectures Including Service on Speakers Bureaus: Penumbra, Comments: Speakers Bureau expenses paid for talks; Patents (Planned, Pending or (ssued): Advanced Catheter Therapies, Comments: devices and methods for perfusion therapy; Patent No. US 8622992 B2; Stock/Stock Options: Penumbra, Comments: hold stock in the company. Tudor G. Jovin-UNRELATED: Consultancy: Codman Neurovascular, Neuravi; Stock/Stock Options: Silk Road, Anaconda, Blockade Medical; Travel/Accommodations/Meeting Expenses Unrelated to Activities Listed: Stryker, Fundació Ictus. Raul G. Nogueira—RELATED: Consulting Fee or Honorarium: Stryker Neurovascular (TREVO-2 Trial, Principal Investigator, modest; DAWN Trial, Principal Investigator, no compensation; TREVO Registry Steering Committee, no compensation); Medtronic (SWIFT Trial Steering Committee, modest; SWIFT-PRIME Trial Steering Committee, no compensation; STAR Trial Angiographic Core Lab, significant), Penumbra (3D Separator Trial Executive Committee, no compensation), Neuravi (ARISE-2 Steering Committee, no compensation), Genentech (Physician Advisory Board, modest), Allm Inc (Physician Advisory Board, no compensation), Editor-In-Chief Interventional Neurology (no compensation). Dileep R. Yavagal—RELATED: Grant: Medtronic, Comments: University of Miami was a site for the SWIFT-PRIME study*; Consulting Fee or Honorarium: Medtronic, Comments: Steering Committee member for this trial; Support for Travel to Meetings for the Study or Other Purposes: Medtronic; UNRELATED: Consultancy: Medtronic; Payment for Lectures Including Service on Speakers Bureaus: Penumbra, Medtronic, Comments: consultant to Medtronic; Travel/Accommodations/Meeting Expenses Unrelated to Activities Listed: Medtronic; Other: Medtronic. Christophe Cognard-UNRELATED: Consultancy: MicroVention, Stryker, Medtronic. Derk D. Purcell—RELATED: Other: BioClinica, Comments: I was a central reader, paid by the imaging clinical research organization (BioClinica); UNRELATED: Consultancy: BioClinica, Comments: central reader for imaging clinical research organization. Reza Jahan-RELATED: Fees for Participation in Review Activities such as Data Monitoring Boards, Statistical Analysis, Endpoint Committees, and the Like: Medtronic, Comments: on Executive Steering Committee of SWIFT PRIME; UNRELATED: Consultancy: Medtronic. Jeffrey L. Saver-RELATED: Grant: Medtronic, Comments: The University of California Regents received payments on the basis of clinical trial contracts for the number of subjects enrolled*; Consulting Fee or Honorarium: Medtronic, Comments: Dr Saver received funding for services as a scientific consultant regarding the SWIFT PRIME trial design and conduct to Medtronic; Support for Travel to Meetings for the Study or Other Purposes: Medtronic, Comments: Dr Saver received funding for travel as a 
scientific consultant regarding the SWIFT PRIME trial design and conduct to Medtronic; UNRELATED: Consultancy: Stryker, Neuravi, BrainsGate, Pfizer, Squibb, Boehringer Ingelheim (prevention only), ZZ Biotech, and St. Jude Medical, Comments: Dr Saver has received funding for services as a scientific consultant regarding trial design and conduct to Stryker, Neuravi, BrainsGate, Pfizer, Squibb, Boehringer Ingelheim (prevention only), ZZ Biotech, and St. Jude Medical; Royalties: University of California, Comments: The University of California has patent rights for retrieval devices for stroke.* Mayank Goyal-RELATED: Grant: Medtronic, Comments: HERMES collaboration, part funding for the ESCAPE trial*; Consulting Fee or Honorarium: Medtronic, Comments: For the design and conduct of the SWIFT PRIME trial; Other: Stryker, MicroVention, Ablynx, Comments: For advice and teaching on acute stroke; UNRELATED: Patents (Planned, Pending or Issued): GE Healthcare, Comments: licensing agreement regarding systems of stroke diagnosis. *Money paid to the institution.

\section{REFERENCES}

1. Hill MD, Demchuk AM, Goyal M, et al; IMS3 Investigators. Alberta Stroke Program Early Computed Tomography Score to select patients for endovascular treatment: Interventional Management of Stroke (IMS)-III trial. Stroke 2014;45:444-49 CrossRef Medline

2. Kidwell CS, Jahan R, Gornbein J, et al; MR RESCUE Investigators. A trial of imaging selection and endovascular treatment for ischemic stroke. N Engl J Med 2013;368:914-23 CrossRef Medline

3. Saver JL, Goyal M, Bonafe A, et al; SWIFT PRIME Investigators. Stent-retriever thrombectomy after intravenous t-PA vs. t-PA alone in stroke. $N$ Engl J Med 2015;372:2285-95 CrossRef Medline

4. Albers GW, Goyal M, Jahan R, et al. Ischemic core and hypoperfusion volumes predict infarct size in SWIFT PRIME. Ann Neurol 2016;79:76-89 CrossRef Medline

5. Albers GW, Goyal M, Jahan R, et al. Relationships between imaging assessments and outcomes in Solitaire With the Intention For Thrombectomy as Primary Endovascular treatment for acute ischemic stroke. Stroke 2015;46:2786-94 CrossRef Medline

6. Saver JL, Goyal M, Bonafe A, et al; SWIFT PRIME Investigators. Solitaire with the Intention for Thrombectomy as Primary Endovascular Treatment for Acute Ischemic Stroke (SWIFT PRIME) trial: protocol for a randomized, controlled, multicenter study comparing the Solitaire revascularization device with IV tPA with IV TPA alone in acute ischemic stroke. Int J Stroke 2015;10:439-48 CrossRef Medline

7. Barber PA, Demchuk AM, Zhang J, et al. Validity and reliability of a quantitative computed tomography score in predicting outcome of hyperacute stroke before thrombolytic therapy: ASPECTS Study
Group-Alberta Stroke Programme Early CT Score. Lancet 2000; 355:1670-74 CrossRef Medline

8. Riedel CH, Zimmermann P, Jensen-Kondering U, et al. The importance of size: successful recanalization by intravenous thrombolysis in acute anterior stroke depends on thrombus length. Stroke 2011;42:1775-77 CrossRef Medline

9. Menon BK, Qazi E, Nambiar V, et al; Interventional Management of Stroke III Investigators. Differential effect of baseline computed tomographic angiography collaterals on clinical outcome in patients enrolled in the Interventional Management of Stroke III trial. Stroke 2015;46:1239-44 CrossRef Medline

10. Hill MD, Demchuk AM, Tomsick TA, et al. Using the baseline CT scan to select acute stroke patients for IV-IA therapy. AJNR Am J Neuroradiol 2006;27:1612-16 Medline

11. Hill MD, Rowley HA, Adler F, et al; PROACT-II Investigators. Selection of acute ischemic stroke patients for intra-arterial thrombolysis with pro-urokinase by using ASPECTS. Stroke 2003;34:1925-31 CrossRef Medline

12. Noorian AR, Rangaraju S, Sun $\mathrm{CH}$, et al. Endovascular therapy in strokes with ASPECTS 5-7 may result in smaller infarcts and better outcomes as compared to medical treatment alone. Interv Neurol 2015;4:30-37 CrossRef Medline

13. Horn CM, Sun CH, Nogueira RG, et al. Endovascular Reperfusion and Cooling in Cerebral Acute Ischemia (ReCCLAIM I). J Neurointerv Surg 2014;6:91-95 CrossRef Medline

14. Leng X, Fang H, Leung TW, et al. Impact of collateral status on successful revascularization in endovascular treatment: a systematic review and meta-analysis. Cerebrovasc Dis 2016;41:27-34 CrossRef Medline

15. Hwang YH, Kang DH, Kim YW, et al. Impact of time-to-reperfusion on outcome in patients with poor collaterals. AJNR Am J Neuroradiol 2015;36:495-500 CrossRef Medline

16. Menon BK, d'Esterre CD, Qazi EM, et al. Multiphase CT angiography: a new tool for the imaging triage of patients with acute ischemic stroke. Radiology 2015;275:510-20 CrossRef Medline

17. Hill MD, Martin RH, Mikulis D, et al. Safety and efficacy of NA-1 in patients with iatrogenic stroke after endovascular aneurysm repair (ENACT): a phase 2, randomised, double-blind, placebo-controlled trial. Lancet Neurol 2012;11:942-50 CrossRef Medline

18. Mishra SM, Dykeman J, Sajobi TT, et al. Early reperfusion rates with IV tPA are determined by CTA clot characteristics. AJNR Am J Neuroradiol 2014;35:2265-72 CrossRef Medline 